\title{
An Unusual Case of Inferior Vena Cava Thrombosis in a Healthy Male Bodybuilder
}

Junichi Imanishi ${ }^{1}$, Michiko Iseri ${ }^{2}$, Masahiro Motoki ${ }^{2}$, Sachiko Yoshikawa ${ }^{1}$, Naohiko Sone ${ }^{1}$, Tomoyuki Honjo ${ }^{1}$, Kohei Kamemura ${ }^{1}$, Kenji Kaihotsu ${ }^{1}$ and Masanori Iwahashi ${ }^{1}$

\begin{abstract}
:
Inferior vena cava (IVC) thrombosis is very rare, particularly in the absence of an apparent congenital caval abnormality or hypercoagulable state. We herein report an unusual case of a healthy and active 62-yearold male bodybuilder with a mass-like IVC thrombus. We placed an IVC filter and began treatment with rivaroxaban. The patient recovered successfully, and the IVC thrombus completely disappeared three months later. This case suggested that extrinsic compression of IVC by a tightened weightlifting belt around the abdomen is a triggering factor of IVC thrombosis, and rivaroxaban, a new oral anticoagulant, may be a useful option for treatment.
\end{abstract}

Key words: inferior vena cava thrombosis, rivaroxaban, anticoagulation, bodybuilder

(Intern Med 57: 2517-2521, 2018)

(DOI: 10.2169/internalmedicine.0377-17)

\section{Introduction}

Inferior vena cava (IVC) thrombosis is an underrecognized entity that is associated with significant morbidity and mortality (1). It is estimated that $2.6-4.0 \%$ patients with lower extremity deep vein thrombosis (DVT) also have IVC thrombosis, whereas thrombus localized only in the IVC is extremely rare (2). IVC thrombosis is commonly associated with outflow obstruction of the IVC, such as that observed with Budd-Chiari syndrome, IVC anomalies, or as a result of external compression by a mass or hematoma (3). Various mechanisms for the origin of this condition have been suggested, particularly in athletes (4). In general, athletes are exposed to many thrombogenic risk factors, including hemoconcentration, trauma, immobilization, longdistance travel, and the use of oral contraceptives, but there is no well-established evidence or treatment (5).

We herein report an unusual case of IVC thrombosis complicated with a pulmonary embolism in a 62-year-old male bodybuilder probably caused by extrinsic compression due to a weightlifting belt.

\section{Case Report}

A 62-year-old man was admitted to our hospital because of worsening shortness of breath over a 2 -week period. His dyspnea began suddenly while riding a bike, and he developed worsening shortness of breath. The patient denied recent trauma, surgery, immobilization, or a family history of thrombotic disorders. His only medication was ibuprofen, when needed. He had no abuse of anabolic steroids and supplements. He was a never-smoker and reported social consumption of alcohol. He reported doing daily physical exercise and had a history of performing excessive bodybuilding exercises using a weightlifting belt to prevent back injury while lifting heavy weights five days a week since his 30 s. He trained one of five parts of his body (legs, chest, back, shoulders and arms) on each day of the week. His workout consisted mainly of a dumbbell or bench presses variation, sometimes with a machine press. His vital signs included blood pressure of $122 / 86 \mathrm{mmHg}$, heart rate of $74 / \mathrm{min}$, and oxygen saturation of $95 \%$ (breathing room air). His body mass index and waist circumference were $25 \mathrm{~kg} / \mathrm{m}^{2}(162 \mathrm{~cm}$, $63 \mathrm{~kg}$ ) and $76 \mathrm{~cm}$, respectively. He had no signs of DVT,

${ }^{1}$ Division of Cardiovascular Medicine, Department of Internal Medicine, Shinko Hospital, Japan and ${ }^{2}$ Department of Clinical Laboratory, Shinko Hospital, Japan

Received: October 19, 2017; Accepted: January 29, 2018; Advance Publication by J-STAGE: April 27, 2018

Correspondence to Dr. Junichi Imanishi, junnnichi@gmail.com 


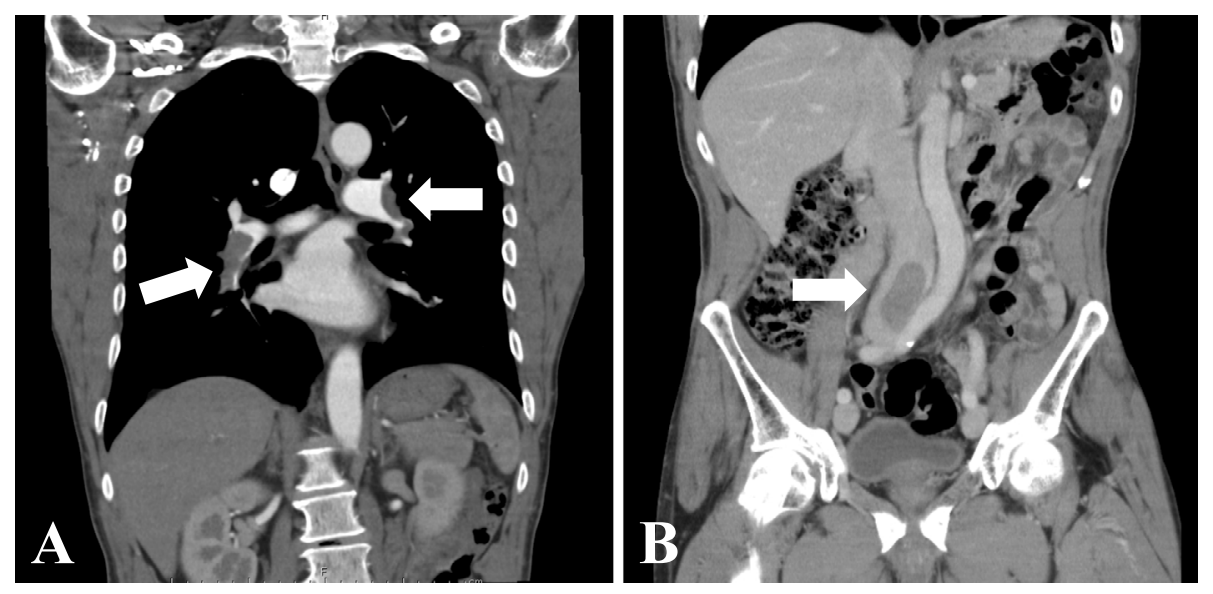

Figure 1. Enhanced computed tomography. Panel A shows the thrombus in the right pulmonary artery and basal segmental branches. Panel B shows a large, mass-like thrombus measuring 50×25 $\mathrm{mm}$ in diameter in the IVC (white arrow). IVC: inferior vena cava
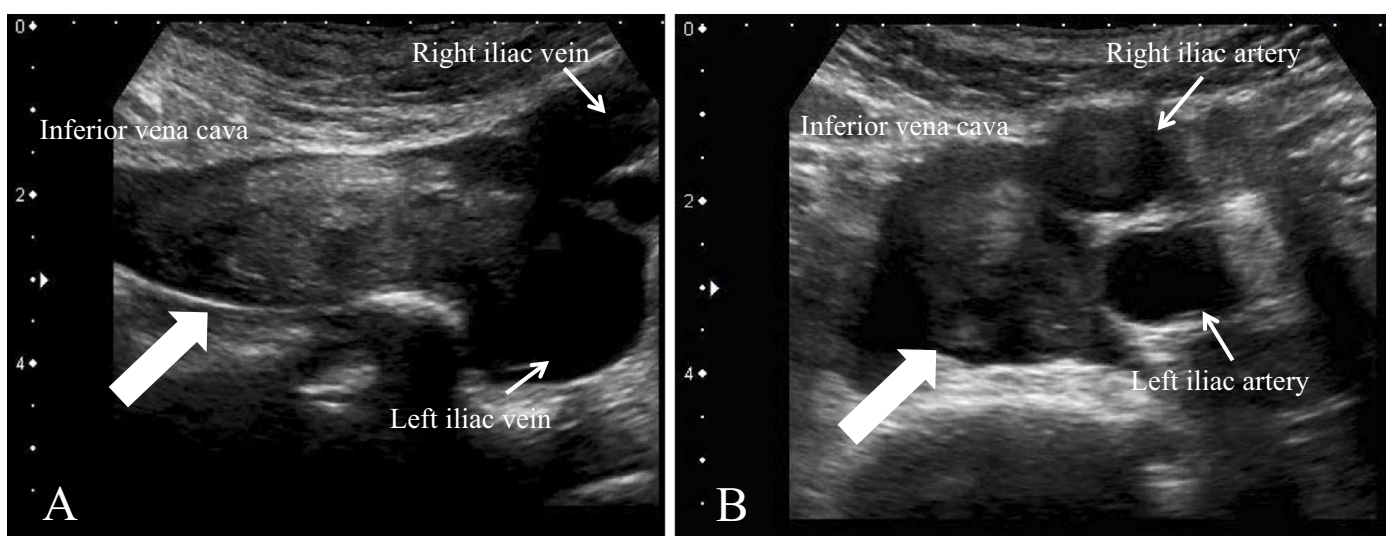

Figure 2. Abdominal ultrasonography showing a free-floating thrombus in the IVC (white arrow) (A: longitudinal axis, B: short axis). IVC: inferior vena cava

such as obvious edema, in his legs. Laboratory findings were ordinary, except for the levels of D-dimer products $(11.8 \mu \mathrm{g} / \mathrm{mL})$. Levels of protein $\mathrm{C}$, protein $\mathrm{S}$, antithrombin III, and antiphospholipid antibodies were within normal limits. An electrocardiogram showed a T-wave inversion in the leads V1-3 and III. However, a transthoracic echocardiogram did not reveal any abnormalities. Contrast-enhanced computed tomography (CT) revealed a thrombus in the right pulmonary artery and basal segmental branches (Fig. 1A). Furthermore, multiplanar reconstruction images revealed a $50 \times 25-\mathrm{mm}$ mass-like thrombus localized at the distal site of IVC (Fig. 1B), and abdominal ultrasonography revealed a free-floating thrombus in IVC (Fig. 2). Contrast-enhanced computed tomography did not show data compatible with malignant pathology or an inflammatory process. Doppler ultrasonography of the lower extremities was performed with no evidence of venous thromboembolism (VTE) visualized.

Because of the vast and floating IVC thrombus at the infra-renal level, we decided to insert an IVC filter in the infra-renal IVC on the first day (before the administration of the anticoagulant). He was subsequently orally administered
$15 \mathrm{mg}$ of rivaroxaban twice daily for 21 days followed by $15 \mathrm{mg}$ daily for maintenance treatment. He responded well to the new treatment with a significant reduction in dyspnea three days after the administration of rivaroxaban and was able to walk up the stairs without shortness of breath. After a month of treatment, follow-up abdominal CT showed a significant reduction in the thrombus, but a small residual thrombus remained. With no evidence of thrombus at the IVC filter, we attempted to remove the IVC filter. However, it proved impossible to snare the hook of the IVC filter at wall because of a tortuous IVC. He continued receiving rivaroxaban at $15 \mathrm{mg}$ daily until the clinical follow-up 3 months after the development of IVC thrombus.

At the 3-month follow-up, venous Doppler imaging of the IVC was performed, and the IVC thrombus had completely disappeared. An ultrasound compression test for the IVC was performed to clarify the impact of the weightlifting belt he used, which demonstrated an easily compressed IVC by the convex probe (Fig. 3). We then replicated situations using the weightlifting belt and not using it in order to examine how the weightlifting belt affected the IVC. Fig. 4 shows a comparison of the spectral Doppler waveforms ob- 


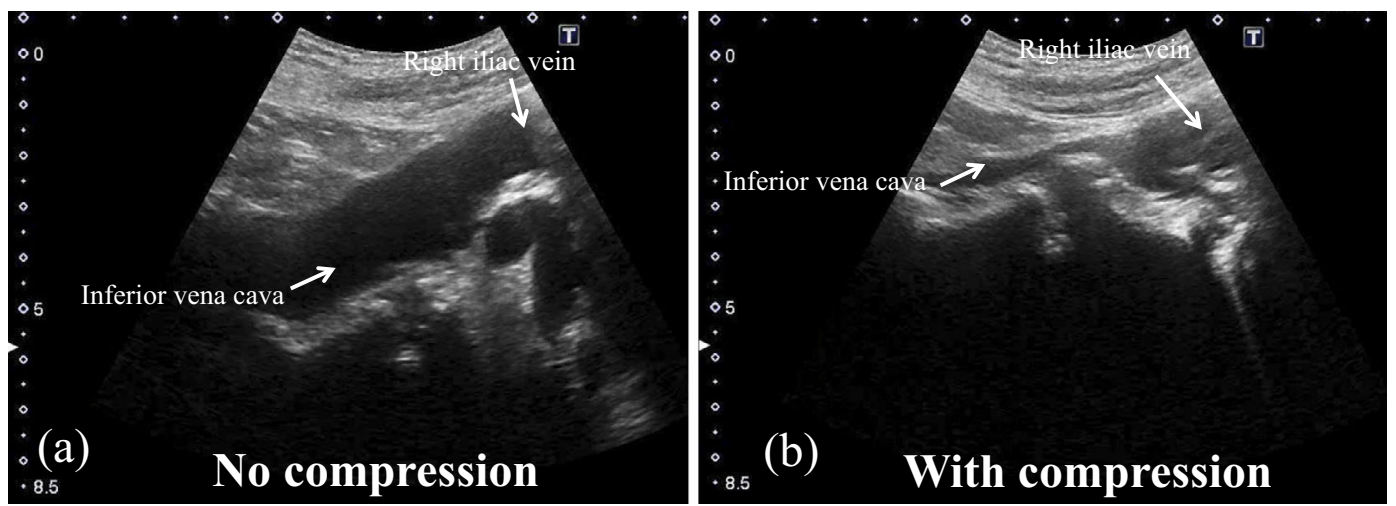

Figure 3. Abdominal ultrasonography after three months of rivaroxaban treatment. An ultrasound compression test for the IVC before (a) and after (b) compression by a convex probe. (b) demonstrates an easily compressed IVC. IVC: inferior vena cava
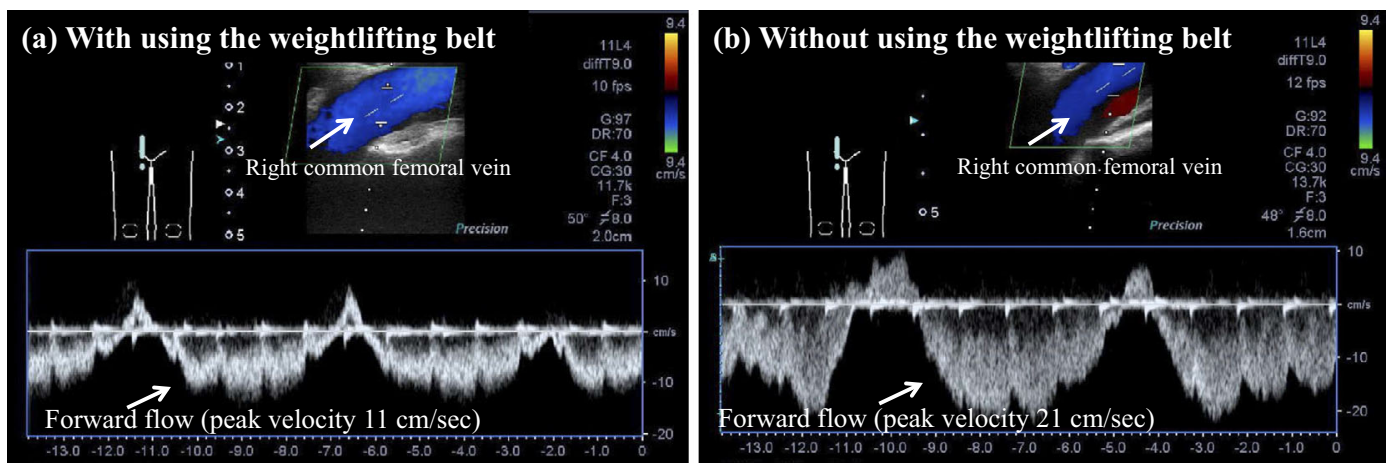

Figure 4. A comparison of the spectral Doppler waveforms obtained at the level of his right common femoral vein with (a) and without (b) the weightlifting belt. (a) demonstrates a more decreased forward flow than without the weightlifting belt $(b)$.

tained at the level of his right common femoral vein with and without the weightlifting belt. When using the weightlifting belt, a more decreased forward flow was observed compared to not using the weightlifting belt, which was an indirect sign of central venous stenosis. He finished a 6month course of rivaroxaban therapy while refraining from using the weightlifting belt, and the IVC thrombus was successfully treated based on the findings of repeated venous Doppler imaging of the IVC, which remained negative for thrombosis (Fig. 5).

\section{Discussion}

Localized thrombosis of IVC is an extremely rare condition in otherwise healthy adults $(2,6)$. Because it is a rare entity, the definitive mechanism of thrombosis and its treatment remain unclear. The patient in our case study had no malignancy, trauma, hematologic abnormality, or a history of surgery. However, data are insufficient to claim that the etiology is "unknown", as this is an extremely rare condition. The risk factors for thromboembolism are well known, and athletes are placed under conditions, such as travel, trauma, immobilization, hemoconcentration, and polycythemia, that can result in exposure to several of these fac- tors (5).

If the pathogenesis of IVC thrombosis in the present case is to be attributed to him being a bodybuilder, some essential aspects need to be considered, including steroid use. Johannesdottir et al. examined the association between the use of glucocorticoids and the risk of VTE and found that current glucocorticoid use is associated with a more than twofold increase in VTE compared with that in non-users (7). In this case, steroid use was not evident, so different mechanisms of bodybuilder-related thrombosis are assumed to have been involved. Another possible cause of the IVC thrombosis in this bodybuilder is the use of a weightlifting belt. Weightlifting belts are commercially marketed with the aim of preventing back injuries while lifting heavy objects. It is thought that they work by helping support and stabilize the spine. However, they may also affect the intra-abdominal pressure while tightening the intra-abdominal organs significantly (8)

Because our patient's IVC was easily compressed by a probe, we considered the mechanism for IVC thrombosis to have been due to his use of a weightlifting belt. Caval flow stasis caused by compression with a weightlifting belt from the outside of the body leads to thrombosis, similar to the so-called venous compression syndromes reported by Butros 
Day1

Insertion of an IVC filter

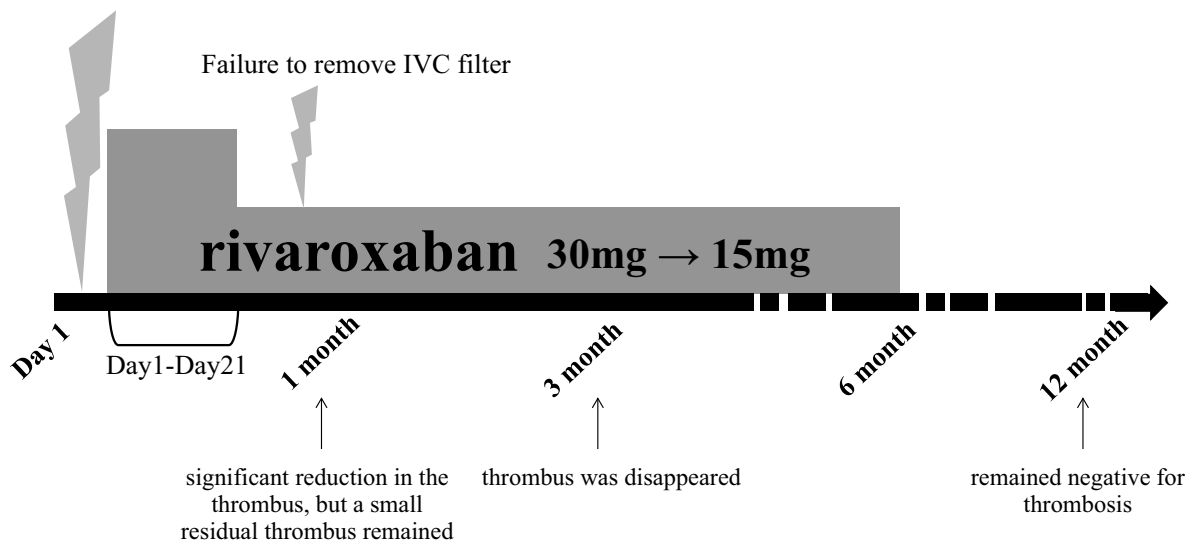

Figure 5. After the insertion of the IVC filter, treatment with oral rivaroxaban for six months resulted in marked improvement in his dyspnea and thrombus. IVC: inferior vena cava

et al. $(9,10)$. Venous compression syndromes are caused by an extrinsic mass effect on a vein by adjacent structures, such as arteries, bones, muscles, or ligaments. In a narrow and confined anatomical space, the otherwise morphologically normal vein becomes trapped between rigid or semirigid surfaces $(11,12)$. In this case, we assumed that the weightlifting belt might compartmentalize IVC combined with his developed inner muscle such as psoas muscle.

Given the rarity of the disease, there is no evidence-based approach for its management. In the present case, the patient had a large floating thrombus in the IVC complicated by a hemodynamically stable PTE. We inserted an IVC filter the day before the administration of a new anticoagulant. We did not opt for the surgical approach, as IVC thrombectomy is an invasive surgery that requires direct caval venotomy or temporary groin arteriovenous fistula creation and does not allow the concomitant treatment of residual stenosis after thrombectomy $(13,14)$. Therefore, this modality has fallen out of favor and is now limited to patients with tumorassociated thrombus $(1,15)$.

Anticoagulants are the gold standard of treatment for patients with IVC thrombosis, but there are no specific recommendations guiding the anticoagulant management in patients. Furthermore, the use of newer anticoagulants remains a challenge. There has only been 1 case report of a 52-yearold man who was successfully treated with rivaroxaban for IVC thrombosis provoked by multiple traumatic injuries (16). The EINSTEIN study showed that oral rivaroxaban was not inferior to warfarin therapy in the treatment of VTE (17). However, the study was based on VTE study data in the general VTE population, and whether or not direct oral anticoagulants (DOACs) are effective options for IVC thrombosis is unclear, as the study did not specify whether patients with IVC thrombosis were included. The advantages of DOACs over warfarin include a lower incidence of significant bleeding, convenience of use, minor drug and food interactions, predictable pharmacokinetics and pharmacodynamic, rapid onset and offset of action, short half-life, and no need for laboratory monitoring (18). Further case series and clinical studies are needed to guide the use of DOACs for IVC thrombosis.

\section{Conclusion}

This case is of interest because IVC thrombosis is rare compared with lower-extremity DVT, particularly localized and mass-like IVC thrombosis. IVC thrombosis has been associated with several factors in the daily life of athletes. Extrinsic compression from outside the body, such as weightlifting belts, may have been an important triggering factor. DOACs with and without an IVC filter may be a useful initial option if a thrombosis is localized in the IVC.

The authors state that they have no Conflict of Interest (COI).

\section{References}

1. McAree BJ, O’Donnell ME, Fitzmaurice GJ, Reid JA, Spence RA, Lee B. Inferior vena cava thrombosis: a review of current practice. Vasc Med 18: 32-43, 2013.

2. Agnelli G, Verso M, Ageno W, et al. The MASTER registry on venous thromboembolism: description of the study cohort. Thromb Res 121: 605-610, 2008.

3. White RH. The epidemiology of venous thromboembolism. Circulation 107: I4-I8, 2003.

4. Grabowski G, Whiteside WK, Kanwisher M. Venous thrombosis in athletes. J Am Acad Orthop Surg 21: 108-117, 2013.

5. Meyering C, Howard T. Hypercoagulability in athletes. Curr Sports Med Rep 3: 77-83, 2004.

6. Farber SP, O'Donnell TF Jr, Deterling RA, Millan VG, Callow $\mathrm{AD}$. The clinical implications of acute thrombosis of the inferior vena cava. Surg Gynecol Obstet 158: 141-144, 1984.

7. Johannesdottir SA, Horvath-Puho E, Dekkers OM, et al. Use of glucocorticoids and risk of venous thromboembolism: a nationwide population-based case-control study. JAMA Intern Med 173: 743-752, 2013.

8. McGill SM, Norman RW, Sharratt MT. The effect of an abdominal belt on trunk muscle activity and intra-abdominal pressure during squat lifts. Ergonomics 33: 147-160, 1990.

9. Campbell DN, Liechty RD, Rutherford RB. Traumatic thrombosis 
of the inferior vena cava. J Trauma 21: 413-415, 1981.

10. Butros SR, Liu R, Oliveira GR, Ganguli S, Kalva S. Venous compression syndromes: clinical features, imaging findings and management. Br J Radiol 86: 20130284, 2013.

11. Eliahou R, Sosna J, Bloom AI. Between a rock and a hard place: clinical and imaging features of vascular compression syndromes. Radiographics 32: E33-E49, 2012.

12. Noorani A, Walsh SR, Cooper DG, Varty K. Entrapment syndromes. Eur J Vasc Endovasc Surg 37: 213-220, 2009.

13. Juhan C, Miltgen G, Barthélémy P, Ayuso D. [Treatment of iliofemoral venous thromboses with surgical thrombectomy]. Bull Acad Natl Med 175: 643-649; discussion 649-650, 1991 (in French).

14. Holper P, Kotelis D, Attigah N, Hyhlik-Durr A, Bockler D. Longterm results after surgical thrombectomy and simultaneous stenting for symptomatic iliofemoral venous thrombosis. Eur J Vasc Endovasc Surg 39: 349-355, 2010.

15. Wang Y, Yuan L, Ge RL, Sun Y, Wei G. Survival benefit of surgical treatment for hepatocellular carcinoma with inferior vena cava/ right atrium tumor thrombus: results of a retrospective cohort study. Ann Surg Oncol 20: 914-922, 2013.

16. Kido K, Noyes E, Gutnik L. Successful use of rivaroxaban in inferior vena cava thrombosis provoked by multiple traumatic injuries and surgeries: A case report. J Clin Pharm Ther 42: 631-633, 2017.

17. Investigators E-P, Buller HR, Prins MH, et al. Oral rivaroxaban for the treatment of symptomatic pulmonary embolism. N Engl J Med 366: 1287-1297, 2012.

18. Mekaj YH, Mekaj AY, Duci SB, Miftari EI. New oral anticoagulants: their advantages and disadvantages compared with vitamin $\mathrm{K}$ antagonists in the prevention and treatment of patients with thromboembolic events. Ther Clin Risk Manag 11: 967-977, 2015.

The Internal Medicine is an Open Access article distributed under the Creative Commons Attribution-NonCommercial-NoDerivatives 4.0 International License. To view the details of this license, please visit (https://creativecommons.org/licenses/ by-nc-nd/4.0/).

(C) 2018 The Japanese Society of Internal Medicine Intern Med 57: 2517-2521, 2018 